\title{
Modelo de implementación de foros virtuales como instrumento para promover aprendizaje colaborativo y desarrollo del pensamiento crítico
}

\author{
Garita González Gabriela ${ }^{1}$ \& Chacón Ballestero Xinia ${ }^{2}$ \\ 1. Encargada del Programa Ingeniería Informática. Escuela de Ciencias Exactas y Naturales. Universidad Estatal a \\ Distancia, Costa Rica. \\ 2. Encargada de Cátedra Sistemas de Calidad en las TIC. Programa Ingeniería Informática. Escuela de Ciencias Exactas \\ y Naturales. Universidad Estatal a Distancia, Costa Rica
}

Recibido: 24 enero 2013 Aceptado: 04 febrero 2013

\begin{abstract}
RESUMEN
El artículo presenta los foros virtuales como una estrategia metodológica que estimula el aprendizaje colaborativo y el pensamiento crítico y ofrece un procedimiento apropiado, paso a paso, para implementar un foro en un curso en línea.
\end{abstract}

Palabras Clave: Formación virtual, Plataformas de aprendizaje, Aprendizaje colaborativo, Foros virtuales.

\begin{abstract}
The article presents the virtual forums as a methodological strategy that stimulates the collaborative learning and de critical thinking and it offers an appropriate step to step procedure to implement a forum on line course.
\end{abstract}

Key words: E-learning, Learning Management System, Collaborative learning, Virtual forums.

Foros como actividad colaborativa y desarrollo del pensamiento crítico

Los foros virtuales se han utilizado en el proceso educativo como estrategia de enseñanza y como instrumento de evaluación de los aprendizajes.

Durante la actividad de los foros los estudiantes llevan a cabo una ardua actividad de trabajo colaborativo y desarrollo del pensamiento crítico, al respecto, Johnson and Johnson, afirman que existe una evidencia real de que los grupos de trabajo colaborativo logran alcanzar niveles más altos de pensamiento y retención de información que los estudiantes que trabajan individualmente. Johnson and Johnson (1986). Koschman argumenta que el término
“Aprendizaje colaborativo" es el modelo de instrucción subyacente y Roschelle\&Teasley lo definen desde una perspectiva más metodológica: "es un mutuo compromiso de los participantes en un esfuerzo coordinado para resolver un problema juntos". (Roschelle \& Teasley, 1995). Por otra parte Arango define el concepto de pensamiento crítico y afirma que este es "el tipo de pensamiento que se caracteriza por manejar y dominar las ideas a partir de su revisión y evaluación para repensar lo que se entiende, se procesa y se comunica. Es un intento activo y sistemático de comprender y evaluar las ideas y argumentos de los otros y los propios. Es concebido como un pensamiento racional, reflexivo e interesado, que decide qué hacer o creer, que es capaz de reconocer y analizar los elementos en sus partes constitutivas". (Arango, 2004). También puede agregarse el estudio de investigación y desarrollo educacional de la universidad de Talca sobre el concepto en cuestión, en donde establece que "el pensamiento crítico se trata de un pensamiento orientado a la comprensión de problemas, la evaluación de alternativas, y la decisión y resolución de los mismos. Pensamiento crítico tiene que ver con comprender, evaluar y resolver". (Universidad de Talca, 2003/2006).

Es por lo expuesto que puede indicarse que la actividad de trabajo colaborativo que se promueve en los foros, fomenta el pensamiento crítico y la comprensión del tema de una manera colectiva, en donde los aportes de cada participante enriquecen la perspectiva de los demás y 
le dan pautas para llegar a una mejor comprensión del tema.

Aspectos a considerar en la Implementación de un foro virtual

A continuación, presentaremos las acciones y criterios a tomar en consideración cuando un profesor desee incluir en sus cursos un foro virtual como parte de sus actividades de formación.

Para definir e implantar un foro virtual como actividad que refuerce el proceso de enseñanzaaprendizaje, se deben considerar:

- ¿Cuándo utilizar un foro?

- ¿Qué se puede discutir en un foro?

- Definición de un lenguaje común.

En adelante se detalla cada aspecto:

1. ¿Cuándo utilizar un foro?

- En la fase inicial, intermedia o final de un curso.

- En un tema de difícil comprensión por parte del estudiante.

- En un tema que demande de las exposición de diferentes experiencias.

- Cuando se desee una mayor profundización y análisis de un tema.

- Cuando se desee realizar una integración de diferentes temas.

- Cuando se desee compartir experiencias individuales

- Se considere necesario construir historias

- Cuando sea necesario realizar una exposición de temas por parte de uno o varios grupos para ser utilizados para realizar análisis del contenido por parte de los otros estudiantes, etc.

2. ¿Qué se puede discutir en el foro?

- Un tema específico relacionado con el contenido del curso.

- Una noticia actualizada

- Una película, documental o anuncio

- Un caso de interés para la materia que se imparte

- Una metodología específica, etc
3. Definición de un lenguaje común

Se debe crear un lenguaje común y unificado en el foro para ello resulta necesario:

- Definir los roles.

- Identificar el foro.

- Definir la normativa para uso del foro.

- Diseñar la rúbrica de evaluación del foro.

Ahora vamos a extender cada una de los aspectos mencionados en el punto 3:

\section{a. Definir los roles}

Se debe definir el rol que cumple cada participante en el foro.

Mediador: es el profesor-tutor a cargo del curso, asegura la mediación pedagógica, informa las reglas, asegura un dialogo respetoso entre los estudiantes, resuelve conflictos, no permite las discusiones negativas cuando se den puntos de vista diferentes. Hace las lecturas de los participantes e indica sugerencias o recomendaciones ante las soluciones brindadas por el grupo;mantiene y promueve la interacción deltema principal, a su vez limita las intervenciones que puedan desviar la finalidad del foro. Durante el periodo del foro motiva la participación, asegurando que los temas desarrollados cumplan con el logro de los propósitos establecidos.

Participantes: son los estudiantes, los cuales investigan y analizan el tema propuesto en el foro, cada participante propone soluciones ante el tema expuesto. Debe hacer lecturas reflexivas, y consultar autores para elaborar párrafos con ideas claras y respaldadas bibliográficamente. Utiliza un lenguaje respetoso ante los puntos de vista de los demás participantes. Cuando un participante hace su aporte al foro, brinda conocimiento y experiencia; de él se espera que realice lecturas de las participaciones hechas por otros estudiantes para realizar intervenciones quecomplementen ideas, apoyen y mejorenlasopiniones que se dan de forma natural. 


\section{b. Identificar el tipo de foro}

Debe definirse para qué será utilizado el foro, para ello se exponen tipos de foros de acuerdo a su función:

- Foro social bienvenida: Es un foro de tipo formativo y social, se sugiere utilizar al inicio del curso. Es un foro donde el profesor y los estudianteshacen una presentación formal con sus datos personales, intereses, profesión y otras características que describen su personalidad, experiencia laboral y aspectos sociales, este foro permite que los integrantes del grupo se conozcan y tengan una idea de los compañeros con los que compartirán durante el desarrollo del curso.

- Foro formativo consultas: Es un foro formativo y técnico, que refuerza el elemento de confiabilidad y disminuye los sentimientos de soledad que puedan experimentar los estudiantes durante las actividades de formación virtuales. Cada participante puede incluir dudas del contenido temático y/o administrativas, el mediador debe definir las reglas de atención de estas consultas y resolver todas las dudas de sus participantes. Se recomienda utilizarlo durante el desarrollo de todo el curso.

- Foro académico sumativo: Es un foro formativo con calificación y porcentaje en la nota final del curso. Desarrolla actividades las cuales se indican en la sección de este documento "que se puede discutir en un foro". Se recomienda que el mismo posea una introducción, cuerpo principal y cierre para la mejora de la comprensión de los participantes. Debe definirse que se le va a evaluar al estudiante a través de una rúbrica de evaluación (el diseño de las mismas se contempla más adelante).

\section{c. Diseñar la normativa para uso de los fo- ros (reglas)}

El foro debe incluir reglas básicas de comunicación, ética y respeto. Éstas deben ser dadas a conocer durante el periodo en que el foro este abierto a las participaciones e interacciones delos estudiantes y del mediador.

Se sugiere que tome en consideración los siguientes aspectos cuando defina las reglas de los foros:

- Sea específico e indique lo que espera de las participaciones del grupo.

- Promueva la lectura diaria del foro. (seguimiento de los temas que se discuten).

- Recomiende hacer intervenciones periódicas e indicar cuantas.

- Recomienda la escritura y comentarios de construcción sencilla, concreta y clara.

- Definir el estilo para el uso de citas bibliográficas.

- Motivar el análisis e investigación, donde se promueva el pensamiento crítico.

- Informar la regulación a ser aplicada, por uso de copias textuales o parciales en las intervenciones.

- Asegurar y promover la utilización de un vocabulario cortés y respetuoso.

\section{d. Diseñar la rúbricas de evaluación del foro}

Las rúbricas facilitan el seguimiento del desempeño delos estudiantes en el foro, representa los criterios que debe cumplir, los estudiantes, con respecto a las participaciones del tema expuesto en el foro. 
TABLA 1

\section{Implementación de un foro}

Actividades propuestas en el documento

Ejemplo de implementación

1. ¿Cuándo utilizar el foro?

Se considera utilizar este foro en la fase final del curso Mercadeo en TIC. En el tema "Responsabilidad social en el Mercadeo", ya que se desea profundizar y analizar, el tema en cuestión, empleando los conocimientos que hasta ahora han alcanzado los estudiantes; además el tema demanda la exposición de diferentes experiencias, por parte de los estudiantes.

2. ¿Qué se puede discutir en un foro?

Para efectos de este foro se utilizó un tema específico relacionado con el contenido del cursoMercadeo en TIC. A saber: Foro No. 2

\section{La responsabilidad Social en el Mercadeo}

3. Definición de un lenguaje común

a. Definir los roles del foro

b. Identificar el tipo de foro

c. Definir la normativa para uso del foro
Estimados estudiantes a continuación se exponen los roles que poseerá cada uno de los participantes del foro; la normativa a seguir por parte de los estudiantes, durante el desarrollo del foro y finalmente la rúbrica con la cual será evaluada su participación en el foro.

Durante este foro los estudiantes se denominan los participantes activos del foro y deben realizar la lectura asignada y las participaciones solicitadas por el profesor-tutor, además debe apegarse a las normativas a seguirse en el foro. El profesor-tutor es el mediador del foro, el cual puede solicitar a los participantes que detalle o amplié sus intervenciones con nuevos argumentos, reflexiones y experiencias. Realizará el cierre respectivo con un resumen de todas las participaciones efectuadas por los estudiantes.

Foro académico sumativo. Valor de $5 \%$ sobre la nota final del curso.

Datos generales:

Objetivo del Foro: Establecer cuál es la responsabilidad social que debe cumplir el mercadeo en su gestión.

Fecha: El foro estará abierto durante una semana, del 5 al 11 de abril.

\section{Instrucciones:}

- Realice la lectura de reflexión: “Existe responsabilidad social en el mercadeo". (Adjunta en la plataforma LMS).

- Las participaciones en el foro deben ser apegados al tema, deben incluir citas de algún autor con sus respectivas referencias.

- No se admiten en el foro copias textuales de libros, internet, revistas u otros no propios que no estén claramente indicados con la cita o referencia. Pueden adjuntar archivos siempre y cuando sean para complementar su participación pero, estos documentos anexos no sustituyen su participación en el foro.

- La participación no debe ser muy extensa, por lo cual deben ser como máximo de media página. Lo anterior para promover discusiones claras y enfocadas en el tema y lograr la agilidad en el desarrollo del mismo.

- Debe velar por participar cuidando la ortografía y redacción de cada una de sus participaciones.

- Para el desarrollo de la conversación es necesario que realice como mínimo tres intervenciones

- Una intervención con su posición respecto al tema.

- Dos intervenciones para interactuar y retroalimentar los aportes de sus compañeros.

- Las intervenciones deben realizarse en días diferentes, para ir participando a lo largo del foro. Cumpliendo: inicio, intermedio y final. Se valorará dicha distribución en el foro.

- Se deben respectar las opiniones y comentarios de cada uno de los participantes y utilizar un vocabulario técnico propio de la signatura.

Instrucciones Específicas:

- $\quad$ Realice la lectura: Fundamente su lectura de lo visto en el Libro de Consulta Kotler, Philip y Gary Armstrong. Marketing. 2009

- Realice sus intervenciones con base en las siguientes interrogantes:

- ¿ ¿Cuál es la relación entre el mercadeo y la responsabilidad social?

- ¿Cuál es el aporte que ofrece el mercado a la responsabilidad social de una empresa? Indique ejemplos de empresas que lo aplican.

- Sus aportes deben evidenciar un análisis crítico, analítico y claro. 
d. Diseñar la rúbrica Esta es la rúbrica utilizada para calificar la participación del estudiante en el foro. de evaluación del foro

Rubrica para evaluar la participación del estudiante en el Foro

\begin{tabular}{|c|c|c|c|c|}
\hline $\begin{array}{l}\text { Aspecto a } \\
\text { evaluar }\end{array}$ & $\begin{array}{l}\text { Excelente } \\
\text { (3 ptos) }\end{array}$ & $\begin{array}{l}\text { Muy bien } \\
\text { (2 ptos) }\end{array}$ & $\begin{array}{l}\text { Necesita mejorar } \\
\quad(1 \text { ptos })\end{array}$ & $\begin{array}{l}\text { No lo realiza } \\
\text { (0 pts) }\end{array}$ \\
\hline Ortografía & $\begin{array}{l}\text { No presenta erro- } \\
\text { res de ortografía. }\end{array}$ & $\begin{array}{l}\text { Presenta } 3 \text { errores } \\
\text { de ortografía }\end{array}$ & $\begin{array}{l}\text { Presenta } 4 \text { errores } \\
\text { ortográficos }\end{array}$ & $\begin{array}{l}\text { Presenta más de } 5 \\
\text { errores ortográficos }\end{array}$ \\
\hline Redacción & $\begin{array}{l}\text { La redacción es } \\
\text { clara, coherente y } \\
\text { se comprende el } \\
\text { texto. }\end{array}$ & $\begin{array}{l}\text { Presenta } 5 \\
\text { problemas en la } \\
\text { redacción lo que } \\
\text { dificulta la lectura }\end{array}$ & $\begin{array}{l}\text { Presenta } 6 \\
\text { problemas en } \\
\text { la redacción } \\
\text { que dificultan la } \\
\text { lectura. }\end{array}$ & $\begin{array}{l}\text { La redacción es } \\
\text { incoherente lo que } \\
\text { no permite una } \\
\text { clara comprensión } \\
\text { del texto. }\end{array}$ \\
\hline $\begin{array}{l}\text { Frecuencia de } \\
\text { participación }\end{array}$ & $\begin{array}{l}\text { Interviene en el } \\
\text { foro } 3 \text { o más veces }\end{array}$ & $\begin{array}{l}\text { Interviene en el } \\
\text { foro al menos } 2 \\
\text { veces. }\end{array}$ & $\begin{array}{l}\text { Interviene en el } \\
\text { foro } 1 \text { vez. }\end{array}$ & $\begin{array}{l}\text { No participa en el } \\
\text { foro. }\end{array}$ \\
\hline $\begin{array}{l}\text { Distribución de } \\
\text { participaciones }\end{array}$ & $\begin{array}{l}\text { Interviene en los } \\
\text { tres momentos } \\
\text { definidos del foro: } \\
\text { inicio, medio y } \\
\text { cierre. }\end{array}$ & $\begin{array}{l}\text { Interviene al } \\
\text { menos en dos de } \\
\text { los momentos del } \\
\text { foro. }\end{array}$ & $\begin{array}{l}\text { Interviene en al } \\
\text { menos uno de los } \\
\text { momentos del } \\
\text { foro. }\end{array}$ & $\begin{array}{l}\text { No interviene en } \\
\text { el foro. }\end{array}$ \\
\hline $\begin{array}{l}\text { Respuesta a } \\
\text { interrogantes }\end{array}$ & $\begin{array}{l}\text { Responden a las } \\
2 \text { interrogan- } \\
\text { tes planteadas } \\
\text { y evidencian } \\
\text { comprensión de } \\
\text { lecturas. }\end{array}$ & $\begin{array}{l}\text { Responde solo a } \\
\text { una de las interro- } \\
\text { gantes planteadas } \\
\text { y su participa- } \\
\text { ción evidencia } \\
\text { comprensión de } \\
\text { lectura. }\end{array}$ & $\begin{array}{l}\text { Responden a am- } \\
\text { bas interrogantes } \\
\text { pero no denota } \\
\text { comprensión } \\
\text { en las lecturas } \\
\text { realizadas. }\end{array}$ & $\begin{array}{l}\text { No responde a } \\
\text { las interrogantes } \\
\text { planteadas. }\end{array}$ \\
\hline Contribuciones & $\begin{array}{l}\text { Aporta al menos } \\
3 \text { contribuciones } \\
\text { adicionales al } \\
\text { foro, tales como } \\
\text { link, documentos } \\
\text { o referencias a } \\
\text { autores. }\end{array}$ & $\begin{array}{l}\text { Aporta al menos } \\
2 \text { contribuciones } \\
\text { al foro, tales como } \\
\text { link, documentos } \\
\text { o referencias a } \\
\text { autores. }\end{array}$ & $\begin{array}{l}\text { Aporta al menos } \\
1 \text { contribución al } \\
\text { foro, tales como } \\
\text { link, documento } \\
\text { o referencia a } \\
\text { autores. }\end{array}$ & $\begin{array}{l}\text { No realiza contribu- } \\
\text { ciones propias, sino } \\
\text { solo comenta la de } \\
\text { sus compañeros }\end{array}$ \\
\hline $\begin{array}{l}\text { Diálogo con } \\
\text { estudiantes y } \\
\text { profesor }\end{array}$ & $\begin{array}{l}\text { Realiza en el foro } \\
\text { al menos } 3 \text { apor- } \\
\text { tes a comentarios } \\
\text { de compañeros } \\
\text { o del tutor y } \\
\text { los mismos son } \\
\text { respetuosos. }\end{array}$ & $\begin{array}{l}\text { Realiza en el foro } \\
2 \text { o menos apor- } \\
\text { tes a comentarios } \\
\text { de los compañe- } \\
\text { ros y del tutor. }\end{array}$ & $\begin{array}{l}\text { Presenta irrespeto } \\
\text { en los comen- } \\
\text { tarios hacia los } \\
\text { compañeros o del } \\
\text { tutor }\end{array}$ & $\begin{array}{l}\text { No dialoga con el } \\
\text { profesor ni con los } \\
\text { participantes. }\end{array}$ \\
\hline Lenguaje Técnic & $\begin{array}{l}\text { Sus participacio- } \\
\text { nes presentan } \\
\text { lenguaje técnico } \\
\text { acorde con el } \\
\text { tema planteado }\end{array}$ & $\begin{array}{l}\text { Sus aportes pre- } \\
\text { sentan lenguaje } \\
\text { técnico en al } \\
\text { menos } 2 \text { de las } \\
\text { participaciones }\end{array}$ & $\begin{array}{l}\text { Sus aportes pre- } \\
\text { sentan lenguaje } \\
\text { técnico en al } \\
\text { menos } 1 \text { de las } \\
\text { participacio-nes. }\end{array}$ & $\begin{array}{l}\text { No utiliza lenguaje } \\
\text { técnico según lo } \\
\text { visto en el curso. }\end{array}$ \\
\hline
\end{tabular}

Fuente propia. 


\section{Encuesta:}

\section{Foro, Responsabilidad social en el Mercadeo}

Para confirmar la asertividad del foro como herramienta metodológica para promover desarrollo del pensamiento crítico y el trabajo colaborativo, se aplicó al 80\% de estudiantes activos en el curso Mercadeo en TIC, pertenecientes a las Licenciaturas de Ingeniería Informática (curso es $100 \%$ en línea), la encuesta denominada: "Encuesta sobre el foro: Responsabilidad social en el Mercadeo", la cual consta de 11 preguntas.

Análisis de resultados

\section{Foros virtuales fomentan el trabajo} colaborativo y el desarrollo del

\section{pensamiento crítico}

En la pregunta No. 1. El 91\% de los estudiantes consideraron que lo discutido y analizado en el foro les contribuyó a conocer más sobre el tema de estudio en contraposición con un $9 \%$ que opinó lo contrario.

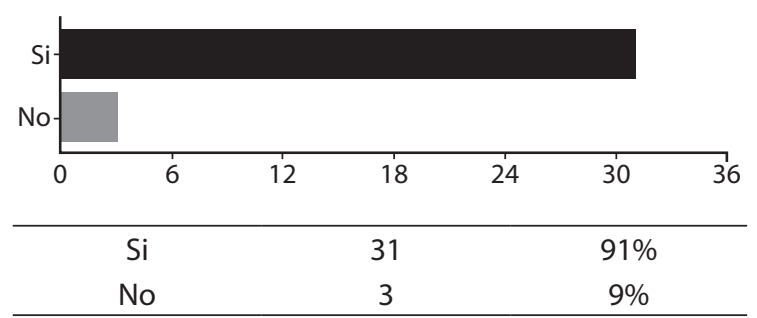

En la pregunta No. 2. El $88 \%$ de los estudiantes consideró que la interacción entre los participantes, influyó en su comprensión y asimilación sobre el tema de estudio a diferencia de un $12 \%$ que no estuvo de acuerdo.

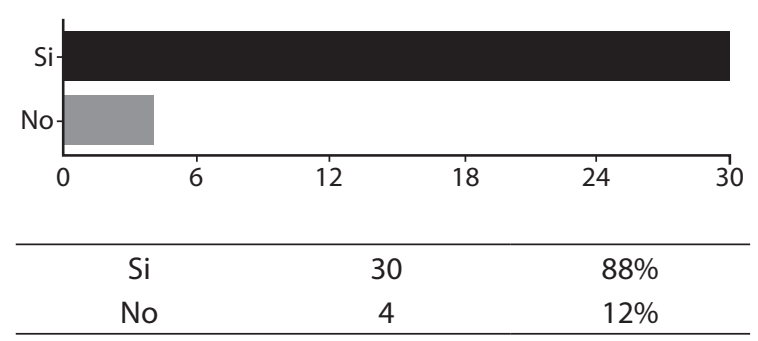

En la pregunta No. 3. El 85\% de los estudiantes consideró que el foro le permitió ampliar su criterio sobre el tema de estudio y en contraposición del $15 \%$ que opinó lo opuesto.

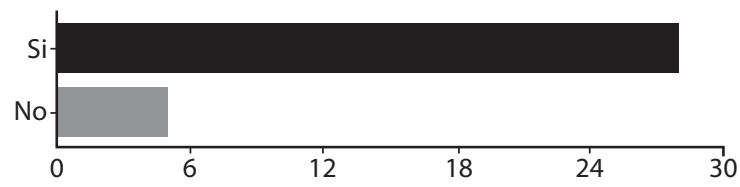

Con respecto a la pregunta No 4. El $79 \%$ de los estudiantes consideraron que el tema analizado en el foro fue enriquecedor para su labor actual o futura en su campo profesional en contraposición a un $21 \%$ que no lo consideró así.

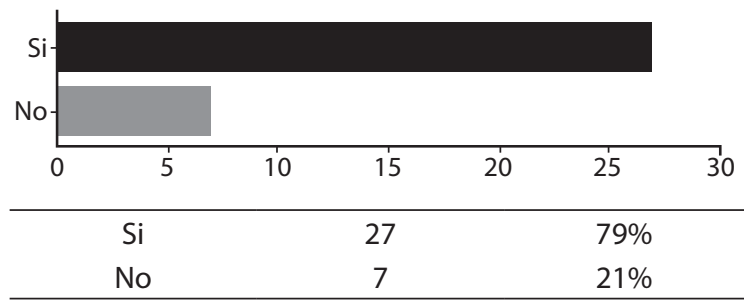

En la pregunta No. 5. El $88 \%$ de los estudiantes consideró que los aportes dados por los compañeros del grupo en el foro, le permitieron generar un aprendizaje colaborativo. En contraposición de un $12 \%$ que no lo consideró de esa manera.

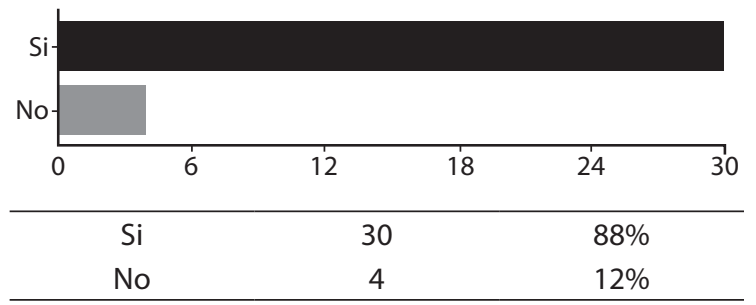

En la pregunta No. 6. El $91 \%$, de los estudiantes considera que la interacción en el foro permite la construcción colectiva de ideas y/o soluciones. Contrario el $9 \%$ que no lo considera así.

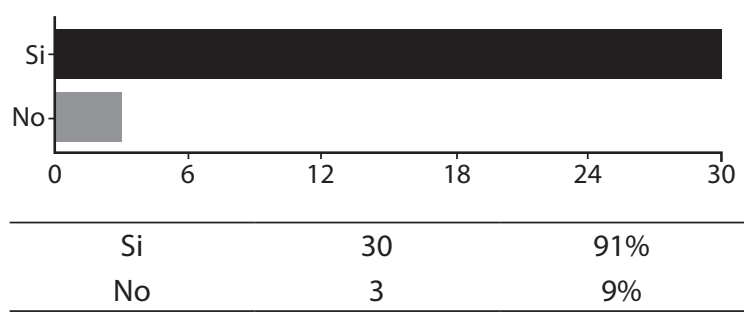


Uso de la herramienta de Foros

dentro de los cursos en línea

En la pregunta No. 7. El 85\% de los estudiantes consideran que los foros debería siempre utilizarse en los cursos en Línea y solo un 15\% no lo considera necesario.

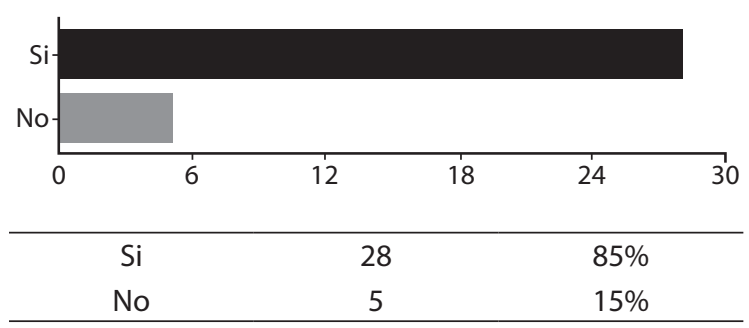

Valor de las intervenciones del tutor dentro del foro

Con respecto a la pregunta No. 8. El 94\% de los estudiantes consideraron que las intervenciones dadas por el tutor sobre su aporte en el foro lo motivaron a continuar participando en contraposición de un $6 \%$ que no lo consideró así.

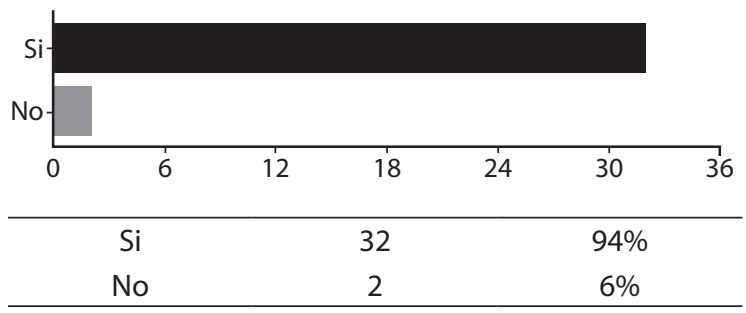

En la pregunta No. 9. El 94\% de los estudiantes consideraron que el tutor es un motivador durante toda la ejecución del foro. En contradicción con un $6 \%$ que opinó diferente.

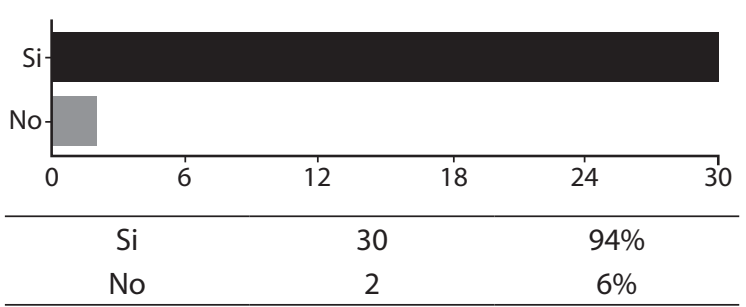

En la pregunta No. 10. El 91\% de los estudiantes opinaron que las intervenciones del tutor propusieron el análisis de los temas, a diferencia de un $9 \%$ que opino lo contrario.

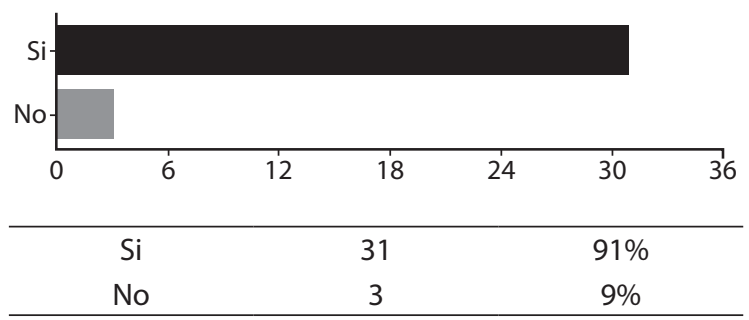

En la pregunta No. 11. El 91\% opinaron que el tutor en el foro, aportó experiencia y conocimiento

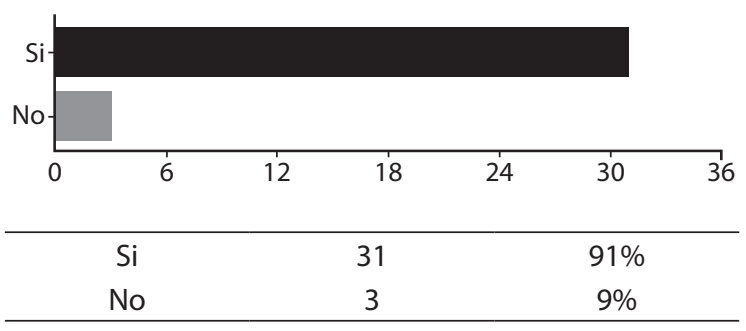

A continuación la tabla No.2. Resumen de encuesta, muestra el porcentaje obtenido de las respuestas dadas por los estudiantes ante las consultas hechas sobre la efectividad del foro; las cuales confirman las afirmaciones presentadas en este artículo. 
TABLA 2

Resumen de encuesta

\begin{tabular}{|c|c|c|c|}
\hline & \multirow{2}{*}{ Pregunta } & \multicolumn{2}{|c|}{ Porcentaje } \\
\hline & & SI & NO \\
\hline 1. & $\begin{array}{l}\text { ¿Considera UD. que lo discutido y analizado en el foro, contribuyó a conocer más sobre el tema } \\
\text { de estudio? }\end{array}$ & 91 & 9 \\
\hline 2. & $\begin{array}{l}\text { ¿Considera UD. que la interacción entre los participantes, influyó en su comprensión y } \\
\text { asimilación sobre el tema analizado? }\end{array}$ & 88 & 12 \\
\hline 3. & $\begin{array}{l}\text { ¿Considera usted que el tema analizado en el foro, fue enriquecedor para su labor actual o } \\
\text { futuro en el campo profesional? }\end{array}$ & 79 & 21 \\
\hline 4. & $\begin{array}{l}\text { ¿Considera UD. que la discusión en el foro, le permitió ampliar su criterio sobre el tema de } \\
\text { estudio? }\end{array}$ & 85 & 15 \\
\hline 5. & $\begin{array}{l}\text { ¿Considera UD. que los aportes dados por los demás compañeros del grupo, durante el foro, } \\
\text { permitieron generar un aprendizaje colaborativo? }\end{array}$ & 88 & 12 \\
\hline 6. & $\begin{array}{l}\text { ¿Considera UD. que la interacción en el foro, permite la construcción colectiva de ideas y/o } \\
\text { soluciones? }\end{array}$ & 91 & 9 \\
\hline 7. & ¿Considera UD. que el foro es una actividad que se debería usar siempre en los cursos en línea? & 85 & 15 \\
\hline 8. & $\begin{array}{l}\text { ¿Considera UD, que las intervenciones dadas por el tutor sobre su aporte en el foro lo } \\
\text { motivaron a continuar participando? }\end{array}$ & 94 & 6 \\
\hline 9. & ¿Considera UD, que el tutor(a) actuó como un motivador(a) durante la ejecución del foro? & 94 & 6 \\
\hline 10. & ¿Considera UD. que las intervenciones efectuadas del tutor, propusieron el análisis del tema? & 91 & 9 \\
\hline 11. & $\begin{array}{l}\text { ¿Considera UD, que las intervenciones efectuadas por el tutor(a), en el foro, aportaron } \\
\text { experiencias y/o conocimiento? }\end{array}$ & 91 & 9 \\
\hline
\end{tabular}

Fuente propia

Como puede observar, el resultado de la encuesta arrojó datos muy acertados acerca del uso de los foros virtuales, ya que se obtuvo casi en un $90 \%$ aprobación por parte de los estudiantes de su uso y beneficios tanto para desarrollo de trabajo colaborativo como para maximizar el pensamiento crítico. Los estudiantes estuvieron de acuerdo en que la herramienta servía apropiadamente para ser utilizada como estrategia de aprendizaje, ya que promovía espacios de discusión y análisis; se pudo obtener que el papel del tutor como moderador y aportador de ideas cumple con un papel fundamental en este proceso.

\section{Conclusiones}

Los foros virtuales resultan ser una estrategia que puede ser empleada en los procesos de enseñanza-aprendizaje en el modelo de educación a distancia apoyada con el uso de las Tecnologías de información y comunicación, a continuación enumeramos los beneficios que se pueden obtener cuando se utiliza un foro virtual de acuerdo a nuestra experiencia:

- Las TIC como apoyo en el proceso de enseñanza-aprendizaje en el modelo de educación a distancia, permite utilizar las herramientas que ofrece Internet, plataformas LMS para el desarrollo de los cursos virtuales.

- Los elementos Internet, plataformas LMS, personas, materias didácticas, mediación pedagógica y actividades se han convertido en una tendencia que refuerza el modelo de educación a distancia.

- El uso de foros virtuales como actividad de un curso permite promover el trabajo colaborativo entre estudiantes-estudiantes, profesores-estudiantes y desarrolla el pensamiento crítico.

- La interacción generada en un foro virtual permite mejorar la comprensión de un tema que es desarrollado y construido de forma 
colectiva. Logrando un mayor nivel de asimilación de los contenidos del curso.

- La mediación pedagógica permite guiar el desarrollo del foro virtual motivando, proponiendo el análisis y aporta experiencias o conocimiento.

- Es ventajoso capacitar a los docentes que desarrollan clases virtuales en el uso de foros virtuales pues promueven la discusión y el pensamiento crítico promoviendo y mejorando las habilidades de análisis y solución de problemas de su entorno profesional y laboral.

- Existe mayor compromiso por parte del estudiante de colaborar en la discusión o exposición del tema.

- Mejora las destrezas y la habilidad de trabajar en equipo entre los estudiantes.

- Permite motivar a los estudiantes a esforzarse por presentar un punto de vista justificado científicamente.

Ante los beneficios presentados instamos a todos los profesionales en docencia que desarrollan cursos en línea a utilizar los foros virtuales como herramienta de apoyo al proceso de formación de sus estudiantes. Igualmente recomendamos a los profesionales en docencia que aún no utilizan cursos virtuales a que integren de forma paulatina foros con actividades formativas con asignación de valor, para mejorar su experiencia en el uso de cursos virtuales; reforzando así la educación a distancia y los procesos de enseñanza-aprendizaje.

\section{Referencias}

Arango, M. (2004). Foros virtuales como estrategia de aprendizaje. Revista Debates Latinoamericanos, $\mathrm{N}^{\mathrm{o}}$ 2. Recuperado de http://www.rlcu.org.ar/revista/ numeros/02-02-Abril-2004/documentos /Arango.pdf
Dominguez, D., y Alonso, L. (2004): "Metodología para el análisis didáctico de foros virtuales". Recuperado de http://edutec2004.lmi.ub.es/pdf/46.pdf

Dillenbourg P.(1999). ¿What do you mean by collaborative learning?. En: Dillenbourg, P.(1999) Collaborative Learning: Cognitive and Computational Approaches. Oxford Elsevier; p 1-19.

Dillenbourg P y Baker, M. (1999). Negotiation spaces in Human collaborative Computer Learning. Proceddings Of the Internatonal Conference on Cooperative Systems (COOP) 6), Juan-Les-Pins(France), June 12141999.

DillenbourgP, .\&Omalley,C. (1996) The evolution of research on collaborative learning. En: Spada\&Reinan (Ed) Learning in Humans and Machines. Towards and interdisciplinary learning Cience (p. 189-211).

DillenbourgP .y Self, J.A. (1992) A computational approach to socially distributed cognition.EuropeanJournal of Psychology of Ed ucation, No.3 (4), 353-372.

Quintero, Nidia. (2011). Orientación del curso Mercadeo en TIC. EUNED. Costa Rica.

Lorenzo García Aretio. (1992). Historia de la educación a distancia. Universidad Nacional de Educación a Distancia, España. Recuperado de http://www.utpl. edu.ec/ried/images/pdfs/vol2-1/historia.pdf

Universidad de Talca, Instituto de Investigación y Desarrollo Educacional, Pensamiento crítico en

la formación universitaria. Documento de Trabajo 2003/6. Proyecto Mecesup TAL 0101. (P. 9-10). Recuperado de http://www.sep.ucr.ac.cr/GESTION/PENSAM1. PDF

Roschelle, J., \&Teasley, S. (1995). The construction of shared knowledge in collaborativeproblem solving.In C. O'Malley (Ed.), Computer-supported collaborative learning (pp.69-197). Berlin, Germany: Springer Verlag. 


\section{Anexos}

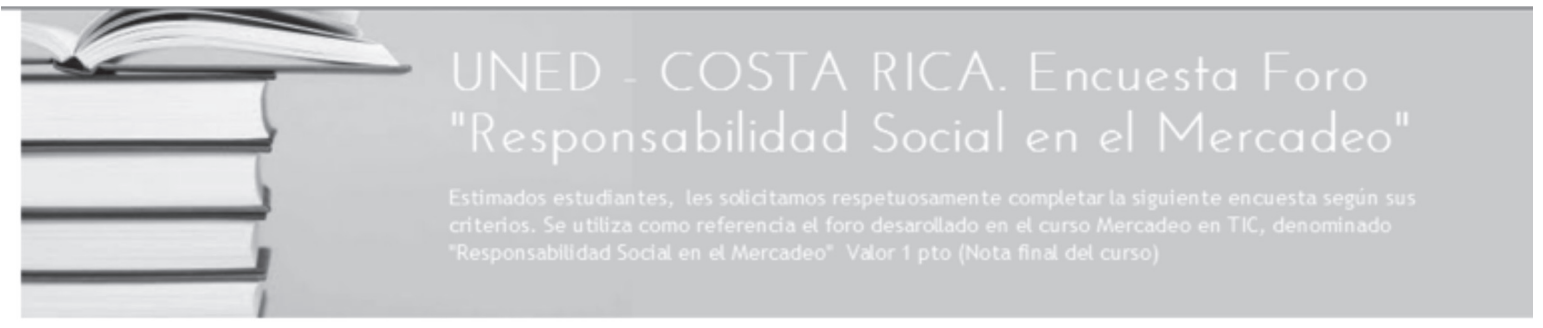

1. ¿Considera UD. que lo discutido y analizado en el foro, contribuyó a conocer más sobre el tema de estudio?

$\square \mathrm{Si}$

No

2. ¿Considera UD. que la interacción entre los participantes, influyó en su comprensión y asimilación sobre el tema analizado?

$\square \mathrm{Si}$

D No

3. ¿Considera UD. que la discusión en el foro, le permitió ampliar su criterio sobre el tema de estudio?

$\square \mathrm{Si}$

No

4. ¿Considera usted que el tema analizado en el foro, fue enriquecedor para su labor actual o futuro en el campo profesional?

$\mathrm{Si}$

No

5. ¿Considera UD. que los aportes dados por los demás compañeros del grupo, durante el foro, permitieron generar un aprendizaje colaborativo?

$\mathrm{Di}$

$\square$ No

6.. ¿Considera UD. que la interaceión en el foro, permite la construceión colectiva de ideas y/o soluciones?

$\square \mathrm{Si}$

$\square$ No

7. ¿Considera UD. que el foro es una actividad que se deberia usar siempre en los cursos en linea?

$\mathrm{Si}$

$\square$ No

8. ¿Considera UD, que las intervenciones dadas por el tutor sobre su aporte en el foro lo motivaron a continuar participando?

$\mathrm{DSi}$

$\square$ No

9. ¿Considera UD, que el tutor(a) actuó como un motivador(a) durante la ejecución del foro?

$\square \mathrm{Si}$

N No

10. ¿Considera UD. que las intervenciones efectuadas del tutor, propusieron el análisis del tema?

$\square \mathrm{Si}$

No

11. ¿Considera UD, que las intervenciones efectuadas por el tutor(a), en el foro, aportaron experiencias y/o conocimiento?

$\square \mathrm{Si}$

$\square$ No 